\title{
«Abilità di comunicazione col paziente? Anche se fosse utile non saprei come cominciare!»
}

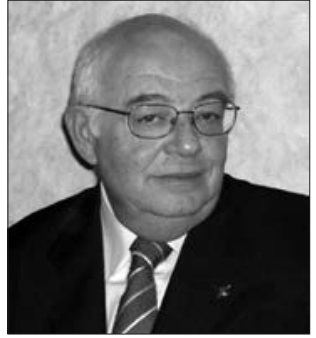

Dr. Bruno Bertone bruno_bertone@virgilio.it

In un reparto di Nefrologia-Dialisi, medici e infermieri sono alle prese con problemi clinici gli uni e con problemi assistenziali gli altri. I carichi di lavoro sono, di norma, poderosi e si può comprendere come, se si propone loro di migliorare le personali abilità di comunicazione, possano pensare: «Ho troppo da fare per pensare anche a come devo parlare con $i$ pazienti». E quindi del tutto comprensibile un atteggiamento almeno scettico nei confronti di: «un'altra cosa ancora da fare». E se, medici e infermieri che pensano in tal modo, avessero ragione? D'altro canto il personale cerca di fornire il miglior servizio di cura e assistenza. Se a questo si aggiunge che i pazienti possono essere di cattivo umore, depressi, arrabbiati col mondo, a volte aggressivi, si può ben comprendere come il desiderio di interagire e comunicare venga meno. Questa visione è, probabilmente, del tutto lecita ma se si dimostrasse che una corretta comunicazione migliora i risultati clinici lo sarebbe ancora?

Se i pazienti traessero comunque sempre beneficio da cure e assistenza la comunicazione avrebbe un ruolo secondario. Nel caso della nefrologia i medici prescrivono le terapie ritenute più confacenti, i dietisti suggeriscono diete adeguate ma i pazienti si adeguano? L'adesione delle persone alla terapia e alla dieta è quella che il personale sanitario auspica e si attende?

L'adesione alle terapie di lungo termine è assai bassa (1) (Figg. 1,2) e i risultati degli studi sulle malattie renali, la dialisi e i trapianti confermano una bassa adesione a terapia e dieta $(2,3)$. Per le terapie di lunga durata siamo intorno a un'adesione del $60 \%$ e per la dieta e l'assunzione di liquidi a meno del $40 \%$ (4).
Se le persone in cura non assumono i farmaci e non si attengono alla dieta significa che fornire le migliori cure e la miglior assistenza non è sufficiente, infatti i pazienti hanno un comportamento non in linea con le attese. A questo punto il problema diviene clinico: il personale sanitario ha l'esigenza di convincere il paziente a seguire le prescrizioni. Il solo modo per convincere qualcuno è parlare, comunicare, negoziare e concordare. Ecco che la comunicazione diventa uno strumento dal quale non si può prescindere.

Ancor prima della comunicazione c'è la disponibilità ad ascoltare e dialogare con il paziente. Un lavoro ha messo in relazione il controllo del diabete e l'empatia di medici che ambulatorialmente seguivano un gruppo di

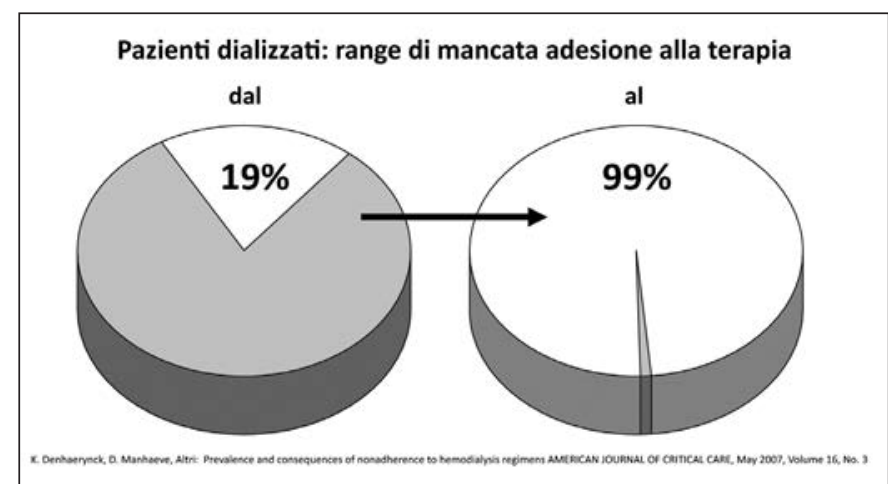

Figura 1.

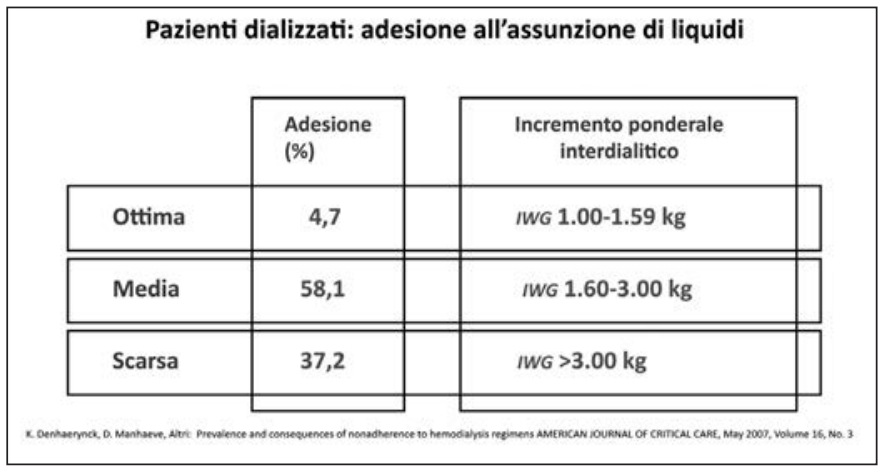

Figura 2. 
891 pazienti (nell'arco di tre anni) $(5,6)$. Ricordiamo che l'empatia può essere definita come: "uguaglianza di sentimenti”. Il medico dialogando con il paziente impiega l'empatia quando gli comunica, anche in modo non verbale, di comprendere i suoi problemi e la sua sofferenza, al paziente è sufficiente essere conscio che il suo interlocutore lo comprende, non vuole che soffra con lui. Nel lavoro citato si sono considerati i valori dell'emoglobina A1c (che esprime lo stato di controllo metabolico del diabete in un periodo corrispondente a 6-8 settimane precedenti il prelievo) e i livelli di LDL-C; si è rilevato che i pazienti dei medici con punteggi di empatia più alti avevano valori HBA1c e LDL-C migliori dei pazienti che facevano riferimento a medici con un più basso punteggio in empatia.

Sembrerebbe incredibile ma l'empatia è stata associata a risultati clinici migliori nel trattamento del diabete! $(5,6)$. Un lavoro in dialisi (7) ha messo a confronto il numero di contatti medico-paziente e i risultati clinici. Sono stati seguiti 678 pazienti, di 76 Centri dialisi no profit, per due anni e sei mesi. Ne è risultato che un più elevato numero di contatti medico-paziente si associa positivamente agli obiettivi di cura.

Un elevato numero di contatti corrisponde anche a più comunicazione e all'instaurarsi di una relazione più stretta tra curante e dializzato.

Si potrebbe obiettare che due soli lavori sono poca cosa, ci sono però altre decine di lavori sulla comunicazione/ relazione e sull'educazione dei pazienti con IRC, prima dell'ingresso in dialisi e durante il periodo della dialisi, nonché per i pazienti in attesa di trapianto di rene. $\mathrm{E}$ sufficiente una ricerca sul web per acquisirne dozzine, naturalmente lanciando la ricerca in lingua inglese, in lingua italiana si trova molto poco. Moltissimi lavori depongono per l'efficacia di comunicazione/relazione ed educazione del paziente. Non resta quindi che l'imbarazzo nella scelta dei lavori scientifici!

Nel contempo si può incominciare a comunicare col paziente, tenendo presente che sarebbe bene:

- impiegare l'empatia,

- favorire l'espressione dei sentimenti,

- non confondersi col paziente,

- consentire al paziente di autogestirsi,

- non attendersi miracolosi cambiamenti di comportamento.

Essere empatici non significa disperarsi col paziente, a lui è sufficiente sentirsi compreso ed accettato come persona. Quando si favorisce l'espressione dei sentimenti, è possibile che il paziente dia sfogo alle proprie emozioni attraverso il pianto ed espressioni di disperazione: «cosa potrò fare?», «... cosa mi succederà?» Fondamentale, per evitare il burnout, sarà l'evitare di farsi carico dei problemi del paziente e non confondersi con lui. La persona malata dovrebbe poter camminare con le proprie gambe ed autogestirsi, i curanti dovrebbero intervenire solo quando ci fosse mancata adesione alla terapia e alla dieta. Quando poi il paziente avesse comportamenti virtuosi non ci si dovrà attendere che questi siano stabili (si veda l'articolo "Una paziente quasi ideale”). I pazienti ricadono spesso in comportamenti poco produttivi per le loro condizioni di salute.

Oltre ad avere queste accortezze si dovrà tenere presente che i pazienti, sino a che non abbiano raggiunto una fase di "accettazione" della loro malattia ("Una paziente quasi ideale"), saranno scarsamente collaborativi, semplicemente perché soggiacciono a un processo psicologico di adattamento che è stressante e di difficile gestione.

Per quanto concerne i dializzati accanto alle fasi del processo psicologico del paziente, descritto nell'articolo "Una paziente quasi ideale", ci sono altre due fasi da considerare, la prima, si verifica, di norma, quando il paziente sia sottoposto a dialisi da poco/pochissimo tempo ed è la fase detta della "luna di miele"(9) (si veda Tabella).

Nel periodo della "luna di miele" i pazienti vivono bene la dialisi che dichiarano essere molto meno disagevole di quanto loro in precedenza descritto. Sono felici per il miglioramento delle personali condizioni di salute conseguenti alla dialisi. In questo periodo le persone si sentono perfettamente in grado di far fronte alla malattia e di accettare i cambiamenti che questa comporta. I pazienti realizzano che gran parte dei loro timori erano infondati e si sentono in grado di poter convivere con la dialisi (9). In seguito i dializzati possono entrare nella fase detta "di transizione" (si veda Tabella) in cui vivono emozioni contrastanti, trascurano le raccomandazioni loro fatte e diffidano della parola dei medici. Spesso si sentono frustrati per il regime dietetico e terapeutico al quale vengono sottoposti. Possono avere sfiducia per le prospettive delle loro condizioni di salute o divenire euforici di fronte a una prospettiva di trapianto. Un qualsiasi cambiamento della terapia li mette in difficoltà e li spaventa (9).

Alle due fasi descritte segue, normalmente, la fase di accettazione nella quale il paziente dà la maggiore disponibilità ed è deciso ad operare positivamente per la sua salute.

Il personale medico ed infermieristico dovrà tenere conto delle fasi psicologiche attraversate dal paziente, si relazionerà impiegando l'empatia, informando, educando alla terapia ed alla dieta. Il successo potrà essere rappresentato da una maggior fiducia del paziente nei confronti di medici e infermieri e da una maggior adesione alle prescrizioni. Medici e infermieri dovranno anche essere consci del fatto che la fase di "accettazione della malattia" non è stabile e che una regressione a fasi precedenti del processo psicologico richiederà ulteriori interventi comunicativi/educativi. 
TABELLA - FASI DI ADATTAMENTO ALLA MALATTIA RENALE CRONICA E CONSIDERAZIONI EDUCAZIONALI

\begin{tabular}{|c|c|c|c|c|}
\hline Stadio & Descrizione & Componente psicosociale & $\begin{array}{l}\text { Considerazioni } \\
\text { per gli educatori }\end{array}$ & Obiettivi formativi \\
\hline Luna di miele & $\begin{array}{l}\text { Il paziente inizia ad } \\
\text { accettare l'idea di avere } \\
\text { un’insufficienza } \\
\text { renale cronica }\end{array}$ & $\begin{array}{l}\text { Convinzione che la dialisi sia } \\
\text { meglio di quanto gli era stato } \\
\text { prospettato }\end{array}$ & $\begin{array}{l}\text { Il paziente può convincersi } \\
\text { di essere un "caso unico" e } \\
\text { trascurare i consigli dei medici }\end{array}$ & $\begin{array}{l}\text { Normalizzare le reazioni } \\
\text { emotive. } \\
\text { Mettere a fuoco i limiti della } \\
\text { dialisi }\end{array}$ \\
\hline Transizione & $\begin{array}{l}\text { Il paziente rinegozia } \\
\text { l'immagine di sé per poter } \\
\text { incorporare la gravità della } \\
\text { propria malattia. }\end{array}$ & $\begin{array}{l}\text { Dolore per la perdita della } \\
\text { salute. } \\
\text { Frustrazione per le limitazioni } \\
\text { che comporta la dialisi. Rabbia. } \\
\text { Senso di colpa. } \\
\text { Depressione/tristezza }\end{array}$ & $\begin{array}{l}\text { Il paziente può avere diffidenza } \\
\text { nei confronti dei medici a } \\
\text { causa delle personali emozioni } \\
\text { contrastanti }\end{array}$ & $\begin{array}{l}\text { Aiutare il paziente ad } \\
\text { identificare le motivazioni } \\
\text { personali verso un certo } \\
\text { obiettivo (dialisi- trapianto) }\end{array}$ \\
\hline Accettazione & $\begin{array}{l}\text { Il paziente } \\
\text { - Accetta la sua condizione } \\
\text { - Fa programmi per il futuro } \\
\text { - Tene comportamenti } \\
\text { positivi }\end{array}$ & $\begin{array}{l}\text { Miglior immagine di sé. } \\
\text { Maggior adesione alla terapia }\end{array}$ & $\begin{array}{l}\text { Il paziente può essere } \\
\text { soddisfatto del trattamento } \\
\text { dialitico }\end{array}$ & $\begin{array}{l}\text { Approvare i cambiamenti } \\
\text { positivi nel comportamento } \\
\text { del paziente }\end{array}$ \\
\hline
\end{tabular}

Modificato da Curtis CE, Rothstein M, Hong BA. Stage-specific educational interventions for patients with end-stage renal disease: psychological and psychiatric considerations.

Educare le persone affette da malattie croniche può portare a risultati molto positivi, non è semplicissimo ma si può fare. L'importante è incominciare, almeno con quelle persone che sembrano essere più disponibili alla relazione. Sarà inevitabile commettere errori che potranno diminuire a mano a mano che si acquisisce esperienza. I primi successi ottenuti saranno di incoraggiamento: il successo genera altro successo e questo sarà la dimostrazione che la comunicazione può divenire uno strumento clinico.

\section{Indirizzo degli Autori:}

Dr. Bruno Bertone

Via Robiolo 119

13825 Valle Mosso (BI)

bruno_bertone@virgilio.it

\section{Bibliografia}

1. WHO: Adherence to long-term therapies: evidence for action, 2003.

2. Denhaerynck K, Manhaeve D, et al. Prevalence and consequences of nonadherence to hemodialysis regimens Am J Crit Care 2007; 16(3).

3. Carter S, Taylor D. A question of choice - compliance in medicine taking - University of London School of Pharmacy.

4. Kugler C, Maeding I, Russell CL. Non-adherence in patients on chronic hemodialysis: an international comparison study 2011 Società Italiana di Nefrologia - ISSN 1121-8428.

5. Ferguson WJ, Candi LM. Culture, language, and the doctorpatient relationship - (2002). Family Medicine and Community Health Publications and Presentations. Paper 61.

6. Hojat M, Louis DZ, et al. Physicians' empathy and clinical outcomes for diabetic patients. Acad Med 2011; 86(3): 359-64.

7. Plantinga LC, Fink NE, et al. Frequency of patient-physician contact in chronic kidney disease care and achievement of clinical performance targets. Int J Qual Health Care 2005; 17(2): 115-21.

8. Curtis CE, Rothstein M, Hong BA. Stage-specific educational interventions for patients with end-stage renal disease: psychological and psychiatric considerations. Progress in Transplantation, 2009; 19(1). 